
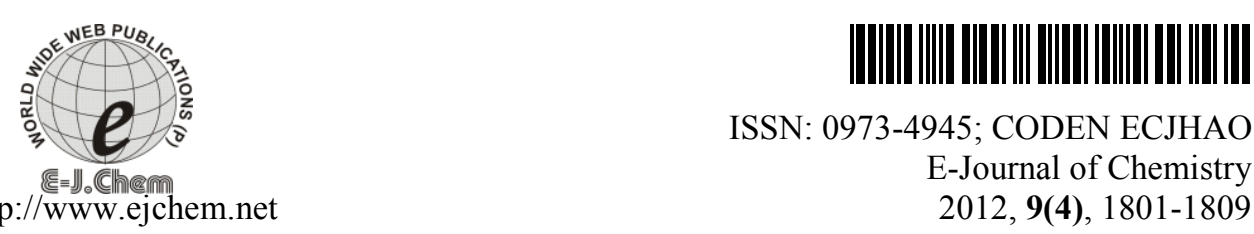

ISSN: 0973-4945; CODEN ECJHAO

E-Journal of Chemistry

http://www.ejchem.net

2012, 9(4), 1801-1809

\title{
Synthesis, Anti-Bacterial and Anti-Fungal Evaluation of Pyrazoline Derivatives
}

\author{
ASHVIN D. PANCHAL AND PRAVINKUMAR M. PATEL* \\ Industrial Chemistry Department, V.P. \& R.P.T.P. Science College, Vallabh Vidyangar- \\ 388120, Gujarat, India \\ ashvin.1985@yahoo.com \\ drpravinpatel@rediffmail.com
}

Received 23 September 2011; Accepted 25 December 2011

\begin{abstract}
The Series of N-(5-(Substituted phenyl)-4,5-dihydro-1H-pyrazol-3yl)-4H-1,2,4-triazol-4-amine compounds were prepared by reaction of 4amino-1,2,4-Triazole with Acetyl Chloride followed by different aromatic aldehydes and cyclization with hydrazine hydrate. The structures of new compounds were confirmed by IR and ${ }^{1} \mathrm{H}-\mathrm{NMR}$ spectral data. Anti-bacterial and Anti-fungal activities were evaluated and compared with the standard drugs, some compounds of the series exhibited promising anti-microbial and anti-fungal activity compared to standard drugs.
\end{abstract}

Keywords: Triazole, Pyrazoline, Antibacterial, Antifungal.

\section{Introduction}

Combat against bacterial infections has resulted in the development of a wide variety of antibiotics. After years of misuse and overuse of antibiotics, bacteria are becoming antibiotic resistant, resulting in a potential global health crisis. There is already evidence that antibacterial resistance is associated with an increase in mortality. Frequently, it is recommended to use new antibacterial agents with enhanced broad-spectrum potency. Therefore, recent efforts have been directed toward exploring novel antibacterial agents ${ }^{1}$. Apart from this, during the past 20 years an increase of invasive fungal infections has been observed, particularly in immunosuppressed patients, which are now causes of morbidity and mortality. However, there is still a critical need for new antifungal agents to treat life threatening invasive mycoses ${ }^{2}$. In order to overcome this rapid development of drug resistance, new agents should preferably consist of chemical characteristics that clearly differ from those of existing agents. In drug designing programs an essential component of the search for new leads is the synthesis of molecules, which are novel yet resemble known biologically active molecules by virtue of the presence of critical structural features. Certain small heterocyclic molecules act as highly functionalized scaffolds and are known pharmacophores of a number of biologically active and medicinally useful molecules ${ }^{3,4}$. 
Electron-rich nitrogen heterocyclics play an important role in diverse biological activities. 2Pyrazoline derivatives have also been reported in the literature to exhibit various pharmacological activities such as antibacterial ${ }^{5-7}$, antidepressant ${ }^{8}$, anticonvulsant ${ }^{9-11}$, antihypertensive $^{12}$, antioxidant ${ }^{13}$, antitumor ${ }^{14}$ and anticancer activities ${ }^{15,16}$. Recently these classes of compounds are reported to possess potential antiviral activity against flavivirus ${ }^{17}$ and $\mathrm{HIV}^{18}$.

1,2,4-triazoles and their heterocyclic derivatives are found to be associated with various biological activities such as anti-inflammatories, CNS stimulants, sedatives, antianxiety compounds, antimicrobial agents ${ }^{19-21}$ and antimycoticones such as fluconazole, intraconazole, voriconazole ${ }^{22,23}$. There are marketed drugs containing the 1,2,4-triazole group, e.g.: Triazolam ${ }^{24}$, Alprazolam ${ }^{25}$, Etizolam $^{26}$ and Furacylin ${ }^{27}$.

In the interest of the above suggestion, we planned to synthesize a system that combines together two biolabile components which are 2-pyrazolines and triazole, to give a compact structure like the title compounds and their possible anti-bacterial and anti-fungal activities. As a part of the continuing effort towards drug discovery, we identified a substituted Pyrazoline (Fig. 1) as potential anti-bacterial and anti-fungal agent. This identification was done on the basis of pharmacophore mapping (Fig. 2) of the Pyrazoline. The pharmacophore model contains ring aromatic (RA), positive ionisable (PI), hydrogen bond acceptor (HBA), hydrogen bond donor (HBD) and aliphatic hydrophobic (HY-ALI) features. Figure 2 shows the mapping of pyrazoline for all the pharmacophoric features except PI. On the basis of pharmacophore mapping, we hypothesized that this type of substituted pyrazolines may show potential bacterial and antifungal activity. In order to validate the hypothesis experimentally, herein we report the activity of pyrazolines derivatives by synthesizing a series of five molecules (4a-e) and evaluating their antibacterial activity against eight microorganism strains of $\mathrm{Gm}^{+\mathrm{ve}}$ as well as $\mathrm{Gm}^{-\mathrm{ve}}$ and antifungal profile against Mucor, Penicillium and Aspergillus fungi. In this study, only the HY-ALI feature associated with ' $\mathrm{B}$ ' ring of the substituted pyrazoline moiety was changed by keeping the basic skeleton intact.

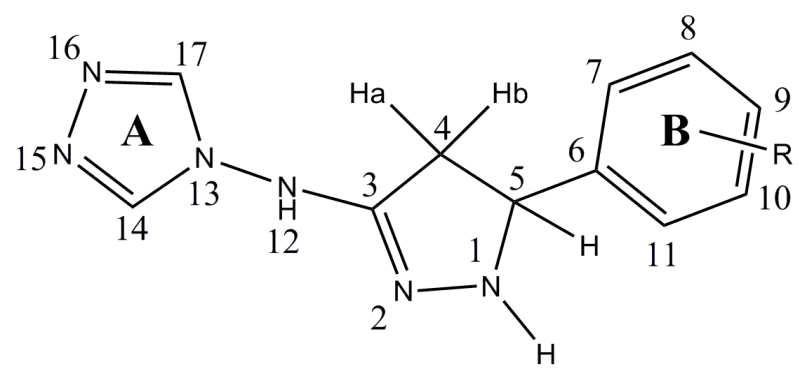

Figure 1

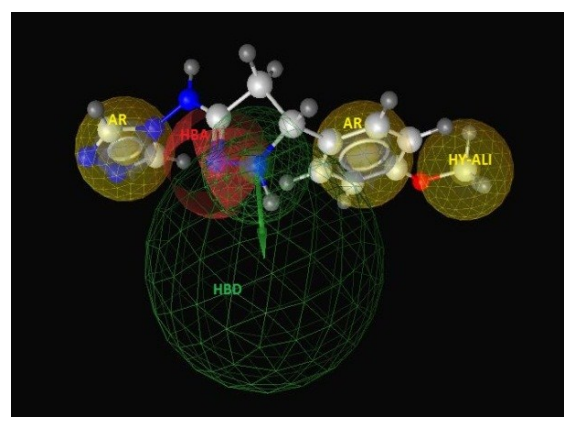

Figure 2 Pharmacophore mapping of Pyrazoline derivative. 
The yellow contour represents the hydrophobic aliphatic feature (HY-ALI), the yellow contour at ring represents ring aromatic feature (AR), the green contour represents hydrogen bond donor feature (HBD) and the red contour represents Hydrogen bond acceptor (HBA).

\section{Experimental Section}

Melting points were determined in open capillary tubes and are uncorrected. All the chemicals and solvents used were of Laboratory Grade and solvents were purified by suitable methods. IR (Infrared spectrum) $\left(\mathrm{KBr}, \mathrm{cm}^{-1}\right)$ were recorded on a Shimadzu-8400 FT-IR spectrometer using $\mathrm{KBr}$ disc, ${ }^{1} \mathrm{H}-\mathrm{NMR}$ spectra were recorded on a Brucker Avance II 400 NMR spectrometer using TMS as an internal standard (chemical shift in $\delta$, ppm) in $\mathrm{CDCl}_{3}$. The homogeneity of the products was checked by TLC using Silica Gel $\mathrm{GF}_{254}$ (E.Merck) and the eluent system was a mixture of Acetone - Pet. ether in 2:8 proportions.

\section{General procedure for the preparation of $\mathrm{N}-(4 \mathrm{H}-1,2,4-t r i a z o l-4-y l)$ acetamide (2)}

$\mathrm{N}-(4 H-1,2,4-$ triazol-4-yl)acetamide was synthesized by acetylation of 4-amino-4H-1,2,4triazole with acetylchloride according to the method reported in the literature ${ }^{28}$. Physical and analytical data are given in tables I.

General procedure for the preparation of 3-(substitutedphenyl)-N-(4H-1,2,4-triazol-4yl)acrylamide $(\mathbf{3 a}-\mathbf{3 e})^{28}$

A solution of $\mathrm{N}-(4 H-1,2,4$-triazol-4-yl)acetamide $(0.01 \mathrm{~mol})$ in absolute ethanol $(50 \mathrm{~mL})$ is refluxed with various aromatic aldehydes in the presence of $2 \% \mathrm{NaOH}(5 \mathrm{ml})$ for $10 \mathrm{~h}$, concentrated, cooled and poured onto ice. The solids thus obtained were recrystallized from appropriate solvents. Physical, analytical and spectroscopic data of compounds are as follows, respectively.

\section{3-(4-chlorophenyl)-N-(4H-1,2,4-triazol-4-yl)acrylamide(3a)}

White crystals,. Yield $74 \%$, m.p. $159^{\circ} \mathrm{C}$; TLC (Acetone : Toluene, $\left.2: 8\right)$. IR: $\left(\mathrm{KBr}, \mathrm{cm}^{-1}\right)$ $3426(\mathrm{~N}-\mathrm{H}), 3123$ (Ar C-H stretch), 2925 and 2852 (C-H stretch), 1691 (NH-C=O), 1653 $(\mathrm{CH}=\mathrm{CH}$ of - Carbonyl- $\mathrm{CH}=\mathrm{CH}-), 1595(\mathrm{C}=\mathrm{N}$ in triazole ring $), 1513(\mathrm{C}=\mathrm{C}$ of aromatic ring), $762(\mathrm{C}-\mathrm{Cl}) .{ }^{1} \mathrm{HNMR}\left(400 \mathrm{MHz}, \mathrm{CDCl}_{3}\right) \delta / \mathrm{ppm}: 8.31$ (s, 1H, N-H), 7.40-7.38 (d, 1H, $\mathrm{CO}-\mathrm{CH}=)$, 7.99-7.97 (d, 1H, =CH-Ar), 7.49-7.47 (d, 2H, Ar-H), 7.84-7.81 (d, 2H, Ar-H), $8.80(\mathrm{ss}, 2 \mathrm{H},-\mathrm{CH}=\mathrm{N}$ in triazole ring).

General procedure for the preparation of N-(5-(substitutedphenyl)-4,5-dihydro-1H-pyrazol3-yl)-4H-1,2,4-triazol-4-amine(4a-e $)^{28}$

To a solution of compound $(3 \mathrm{a}-3 \mathrm{e})(0.02 \mathrm{~mol})$ and $99 \%$ hydrazine hydrate $(0.04 \mathrm{~mol})$ in absolute ethanol and add few drops of hydrochloric acid. The reaction mixtures were refluxed for $8-10 \mathrm{~h}$, distilled in vacuum and cooled. The separated solids were filtered, washed with ether and recrystallized from appropriate solvents. Physical, analytical and spectroscopic data of compounds $(4 \mathrm{a}-4 \mathrm{e})$ are as follows, respectively.

\section{$\mathrm{N}$-(5-(4-chlorophenyl)-4,5-dihydro-1H-pyrazol-3-yl)-4H-1,2,4-triazol-4-amine(4a)}

Yellowish crystals, Yield $60 \%$, m.p. $198^{\circ} \mathrm{C}$; TLC (Acetone : Pet.ether, $(2: 8)$. IR: $\left(\mathrm{KBr}, \mathrm{cm}^{-1}\right)$ $3110(\mathrm{~N}-\mathrm{H}), 3070(\mathrm{Ar} \mathrm{CH}$ stretch), 2985 and $2930(\mathrm{C}-\mathrm{H}$ stretch $), 1630(\mathrm{C}=\mathrm{N})$ in pyrazoline ring, $1580(\mathrm{~N}-\mathrm{N})$ in pyrazoline ring, $1380(\mathrm{C}-\mathrm{N}) .{ }^{1} \mathrm{H}$ NMR $\left(400 \mathrm{MHz}, \mathrm{CDCl}_{3}\right) \delta / \mathrm{ppm}: 7.45$ 
(d, 2H, Ar-H), 7.79 (d, 2H, Ar-H), (s, 1H, -NH- missing), 7.26 (s, 1H, NH in pyrazoline ring), 7.78-7.76 (dd, $2 \mathrm{H}, \mathrm{CH}_{2}$ in pyrazoline ring), $8.60(\mathrm{~s}, 2 \mathrm{H}, \mathrm{CH}=\mathrm{N}$ in triazole ring).

$\mathrm{N}$-(5-(4-(dimethylamino)phenyl)-4,5-dihydro-1H-pyrazol-3-yl)-4H-1,2,4-triazol-4-amine(4b) Yellowish crystals, Yield 50\%, m.p. $254^{\circ} \mathrm{C}$; TLC (Acetone : Pet.ether, $(2: 8)$. IR: $\left(\mathrm{KBr}, \mathrm{cm}^{-1}\right)$ $3410(\mathrm{~N}-\mathrm{H}), 3060(\mathrm{Ar} \mathrm{CH}$ stretch$), 2915$ and $2840(\mathrm{C}-\mathrm{H}$ stretch $), 1610(\mathrm{C}=\mathrm{N})$ in pyrazoline ring, $1520(\mathrm{~N}-\mathrm{N})$ in pyrazoline ring, $1360(\mathrm{C}-\mathrm{N}) .{ }^{1} \mathrm{H} \mathrm{NMR}\left(400 \mathrm{MHz}, \mathrm{CDCl}_{3}\right) \delta / \mathrm{ppm}: 7.69$ (d, 2H, Ar-H), 7.71 (d, 2H, Ar-H), (s, 1H, -NH- missing), 7.25 (s, 1H, NH in pyrazoline ring), 6.72-6.70 (dd, $2 \mathrm{H}, \mathrm{CH}_{2}$ in pyrazoline ring), $8.58(\mathrm{~s}, 2 \mathrm{H}, \mathrm{CH}=\mathrm{N}$ in triazole ring), 3.02 $\left(\mathrm{s}, 6 \mathrm{H}, \mathrm{N}-\left(\mathrm{CH}_{3}\right)_{2}\right)$.

\section{$\mathrm{N}$-(5-(naphthalen-1-yl)-4,5-dihydro-1H-pyrazol-3-yl)-4H-1,2,4-triazol-4-amine(4c)}

Yellowish crystals, Yield 55\%, m.p. $>300^{\circ} \mathrm{C}$; TLC (Acetone : Pet.ether, (2:8). IR: $\left(\mathrm{KBr}, \mathrm{cm}^{-}\right.$ 1) $3420(\mathrm{~N}-\mathrm{H}), 3080(\mathrm{Ar} \mathrm{CH}$ stretch), 2940 and $2860(\mathrm{C}-\mathrm{H}$ stretch $), 1580(\mathrm{C}=\mathrm{N})$ in pyrazoline ring, $1510(\mathrm{~N}-\mathrm{N})$ in pyrazoline ring, $1325(\mathrm{C}-\mathrm{N}) .{ }^{1} \mathrm{H}$ NMR $\left(400 \mathrm{MHz}, \mathrm{CDCl}_{3}\right)$

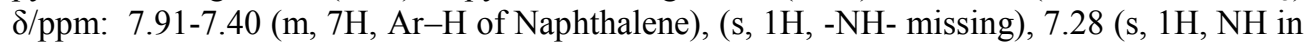
pyrazoline ring), 7.73-7.71 (dd, $2 \mathrm{H}, \mathrm{CH}_{2}$ in pyrazoline ring), $9.68(\mathrm{~s}, 2 \mathrm{H}, \mathrm{CH}=\mathrm{N}$ in triazole ring).

\section{N-(5-(3-methoxyphenyl)-4,5-dihydro-1H-pyrazol-3-yl)-4H-1,2,4-triazol-4-amine(4d)}

Greenish crystals, Yield 58\%, m.p. $162^{\circ} \mathrm{C}$; TLC (Acetone : Pet.ether, $(2: 8)$. IR: $\left(\mathrm{KBr}, \mathrm{cm}^{-1}\right)$ $3140(\mathrm{~N}-\mathrm{H}), 3020($ Ar CH stretch), 2910 and $2830(\mathrm{C}-\mathrm{H}$ stretch $), 1600(\mathrm{C}=\mathrm{N})$ in pyrazoline ring, $1515(\mathrm{~N}-\mathrm{N})$ in pyrazoline ring, $1300(\mathrm{C}-\mathrm{N}) .{ }^{1} \mathrm{H}$ NMR $\left(400 \mathrm{MHz}, \mathrm{CDCl}_{3}\right) \delta / \mathrm{ppm}^{\circ}$ 6.976.94 (d, 2H, Ar-H), 7.77 (d, 1H, Ar-H), 7.80 (d, 1H, Ar-H), (s, 1H, -NH- missing), 7.25 (s, $1 \mathrm{H}, \mathrm{NH}$ in pyrazoline ring), 7.78-7.79 $\left(\mathrm{dd}, 2 \mathrm{H}, \mathrm{CH}_{2}\right.$ in pyrazoline ring), $8.62(\mathrm{~s}, 2 \mathrm{H}, \mathrm{CH}=\mathrm{N}$ in triazole ring), $3.85\left(\mathrm{~s}, 3 \mathrm{H}, \mathrm{Ar}-\mathrm{OCH}_{3}\right)$.

\section{2-(3-(4H-1,2,4-triazol-4-ylamino)-4,5-dihydro-1H-pyrazol-5-yl)phenol(4e)}

Shiny greenish crystals, Yield $54 \%$, m.p. $214^{\circ} \mathrm{C}$; TLC (Acetone : Pet.ether, (2:8). IR: (KBr, $\left.\mathrm{cm}^{-1}\right) 3440(\mathrm{~N}-\mathrm{H}), 3010$ (Ar CH stretch), 2970 and $2860(\mathrm{C}-\mathrm{H}$ stretch), $1620(\mathrm{C}=\mathrm{N})$ in pyrazoline ring, $1570(\mathrm{~N}-\mathrm{N})$ in pyrazoline ring, $1360(\mathrm{C}-\mathrm{N}), 1260,1040(\mathrm{C}-\mathrm{O}) .{ }^{1} \mathrm{H}$ NMR (400 MHz, $\left.\mathrm{CDCl}_{3}\right) \delta / \mathrm{ppm}$ : 7.41-6.95 (m, 4H, Ar-H), (s, 1H, -NH- missing), $7.30(\mathrm{~s}, 1 \mathrm{H}$, $\mathrm{NH}$ in pyrazoline ring), 7.34-7.32 (dd, $2 \mathrm{H}, \mathrm{CH}_{2}$ in pyrazoline ring), $8.71(\mathrm{~s}, 2 \mathrm{H}, \mathrm{CH}=\mathrm{N}$ in triazole ring), $11.38(\mathrm{~s}, 1 \mathrm{H}, \mathrm{Ar}-\mathrm{OH})$.

\section{Result and Discussion}

\section{Chemistry}

Factors such as the structure and position of the substituents have profoundly influenced the rate of the reaction. The generally accepted interpretation of this reaction, involves the initial formation of an aryl hydrazone with subsequent nucleophilic attack of nitrogen upon the carbon-carbon double bond at $\beta$ position. Hence the electropositive nature of $\beta$ carbon may control the overall rate of the reaction. The electropositive nature of $\beta$ carbon is controlled by the aromatic ring directly connected to it. Halogens being electron withdrawing in nature significantly increase the positive character of $\beta$ carbon lead to faster reaction while electron donating alkyl and alkoxy groups contributed for slower reaction. 


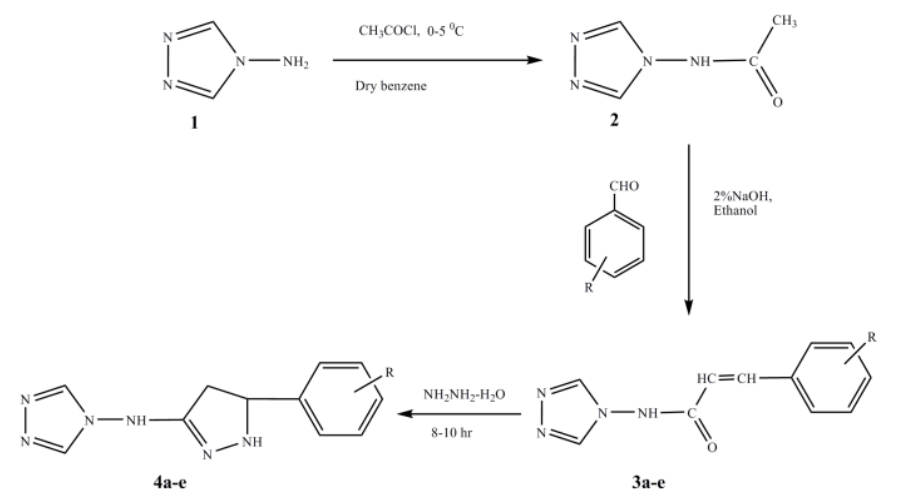

Scheme 1 Synthesis of N-(5-(substitutedphenyl)-4,5-dihydro-1H-pyrazol-3-yl)-4H-1,2,4triazol-4-amine 4a-e.

Table 1 - Physical data of synthesized compounds 2, 3a-e and 4a-e.

\begin{tabular}{|c|c|c|c|c|c|c|}
\hline Compound & $\mathbf{R}$ & $\begin{array}{c}\text { M.P. } \\
\mathbf{( \mathbf { C } )}\end{array}$ & $\begin{array}{c}\text { Yield } \\
\mathbf{( \% )}\end{array}$ & $\begin{array}{c}\text { Mol. } \\
\text { Formula }\end{array}$ & $\begin{array}{c}\text { Mol. } \\
\text { Weight }\end{array}$ & $\begin{array}{c}\text { Recrystallization } \\
\text { solvent* }\end{array}$ \\
\hline $\mathbf{2}$ & - & 155 & 84 & $\mathrm{C}_{4} \mathrm{H}_{6} \mathrm{~N}_{4} \mathrm{O}$ & 126.12 & 1 \\
\hline 3a & $4-\mathrm{Cl}$ & 159 & 74 & $\mathrm{C}_{11} \mathrm{H}_{9} \mathrm{ClN}_{4} \mathrm{O}$ & 248.67 & 1 \\
\hline 3b & $4-\mathrm{N}\left(\mathrm{CH}_{3}\right)$ & 186 & 70 & $\mathrm{C}_{13} \mathrm{H}_{15} \mathrm{~N}_{5} \mathrm{O}$ & 257.29 & 2 \\
\hline 3c & Naphthalene & 225 & 69 & $\mathrm{C}_{15} \mathrm{H}_{12} \mathrm{~N}_{4} \mathrm{O}$ & 264.28 & 3 \\
\hline 3d & $3-\mathrm{OCH}$ & 126 & 72 & $\mathrm{C}_{12} \mathrm{H}_{12} \mathrm{~N}_{4} \mathrm{O}_{2}$ & 244.25 & 1 \\
\hline 3e & $2-\mathrm{OH}$ & 196 & 65 & $\mathrm{C}_{11} \mathrm{H}_{10} \mathrm{~N}_{4} \mathrm{O}_{2}$ & 230.22 & 2 \\
\hline 4a & $4-\mathrm{Cl}$ & 198 & 60 & $\mathrm{C}_{11} \mathrm{H}_{11} \mathrm{ClN}_{6}$ & 262.70 & 2 \\
\hline 4b & $4-\mathrm{N}\left(\mathrm{CH}_{3}\right)$ & 254 & 50 & $\mathrm{C}_{13} \mathrm{H}_{17} \mathrm{~N}_{7}$ & 271.32 & 2 \\
\hline 4c & Naphthalene & $>300$ & 55 & $\mathrm{C}_{15} \mathrm{H}_{14} \mathrm{~N}_{6}$ & 278.31 & 5 \\
\hline 4d & $3-\mathrm{OCH}$ & 162 & 58 & $\mathrm{C}_{12} \mathrm{H}_{14} \mathrm{~N}_{6} \mathrm{O}$ & 258.28 & 2 \\
\hline 4e & $2-\mathrm{OH}$ & 214 & 54 & $\mathrm{C}_{11} \mathrm{H}_{12} \mathrm{~N}_{6} \mathrm{O}$ & 244.25 & 4 \\
\hline
\end{tabular}

*1. Methanol, 2. Ethanol, 3. Acetone, 4. Methanol-Water, 5. Ethanol-Water.

Structures of compounds 4a-e were confirmed by IR and ${ }^{1} \mathrm{H}$ NMR spectroscopic techniques. All of the Pyrazoline possesses similar basic skeletal structure. Proton NMR signals were assigned by comparing the spectra of the products $(4 \mathrm{a}-\mathrm{e})$ with their corresponding chalcones.

Pharmacological results:

Antibacterial activity

All the synthesized compounds were screened for their in vitro antibacterial activity. Bacillus megaterium, Bacillus subtilus, Micrococcus luteus, Staphylococcus aureus, Eschericha coli, Enterobacter, Proteus vulgaris and Pseudomonas aeruginosastrains were used to determine antibacterial activity in which first four are gram positive bacteria while later four are gram negative bacteria. Antibacterial activities of all samples were screened by the agar well diffusion method ${ }^{29,30}$. Compounds $4 \mathrm{~b}, 4 \mathrm{~d}$ and $4 \mathrm{e}$ were most potent and comparable to activities of standard antibiotic chloramphenicol against Bacillus megaterium, Micrococcus luteus Staphylococcus, Proteus vulgaris and Enterobacter. Weak activity was observed with the other compound $4 \mathrm{a}$ and $4 \mathrm{c}$. 
Table 2: Antibacterial activity of Chalcones and Pyrazoline derivatives.

\begin{tabular}{|c|c|c|c|c|c|c|c|c|}
\hline \multicolumn{9}{|c|}{ Antimicrobial Activity of Synthetic Compound (In mm) } \\
\hline \multirow[b]{2}{*}{ Compounds } & \multicolumn{8}{|c|}{ Organisms } \\
\hline & $\mathbf{A}$ & B & $\mathbf{C}$ & $\mathbf{D}$ & $\mathbf{E}$ & $\mathbf{F}$ & G & $\mathbf{H}$ \\
\hline Chloramphenicol & +++ & +++ & +++ & +++ & +++ & ++ & ++ & ++ \\
\hline 3a & - & - & - & - & - & - & - & - \\
\hline 3b & ++ & - & ++ & - & - & - & - & - \\
\hline $3 \mathrm{c}$ & - & - & - & - & - & - & - & - \\
\hline 3d & ++ & - & - & - & - & - & - & - \\
\hline $3 e$ & - & - & ++ & - & - & ++ & - & - \\
\hline $4 a$ & - & - & - & - & - & - & - & - \\
\hline $4 b$ & +++ & - & +++ & - & - & - & - & - \\
\hline $4 \mathrm{c}$ & - & - & - & - & - & - & - & - \\
\hline 4d & +++ & - & - & ++ & - & - & +++ & - \\
\hline $4 e$ & - & - & - & - & - & +++ & ++ & - \\
\hline \multicolumn{9}{|c|}{$\begin{array}{c}\text { A: Bacillus megaterium, B: Bacillus subtilus, C: Micrococcusluteus, D: } \\
\text { Staphylococcus aureus, E: Eschericha coli, F: Enterobacter, G: Proteus } \\
\text { vulgaris, H: Pseudomonas aeruginosa }\end{array}$} \\
\hline \multicolumn{9}{|c|}{$\begin{array}{c}+:<6 \mathrm{~mm}(\text { poor }),++:<12 \mathrm{~mm}(\text { good) },+++:<18 \mathrm{~mm}(\mathrm{v} . g o o d),-: \text { Without } \\
\text { activity }\end{array}$} \\
\hline
\end{tabular}
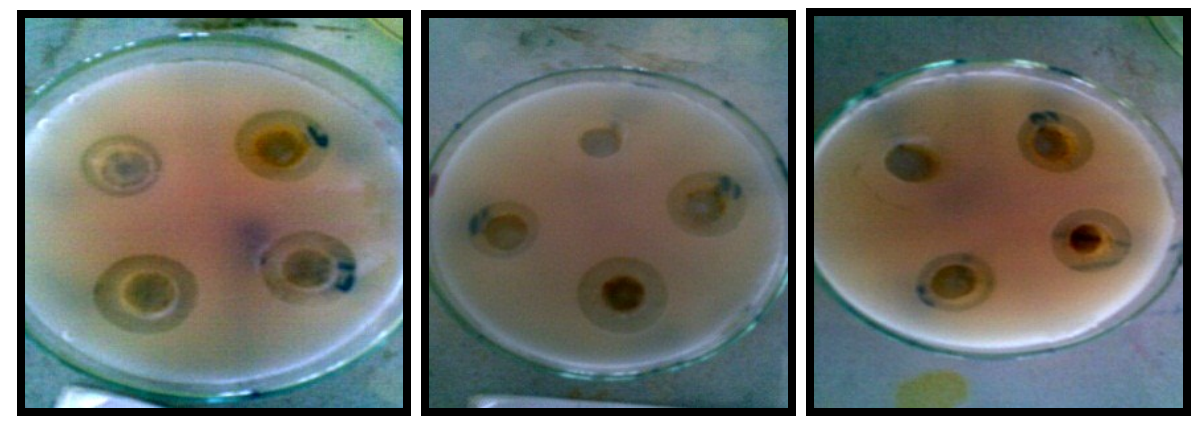

Figure 3: antibacterial activity of Pyrazoline derivatives.

Antifungal activity

All the synthesized compounds were also screened for their in vitro antifungal activity against Mucor, A. niger and Penicillium strains. The zone of inhibition was measured in millimeters. Antifungal activities of all compounds were screened by the turbidometry method $^{31}$. Activity of extract was compared with standard antibiotics fluconazole fungi. DMSO was used as solvent. All compounds are active against Mucor, A. niger and penicillium. Compounds $4 \mathrm{~b}, 4 \mathrm{c}$ and $4 \mathrm{e}$ provided the best antifungal activity and compared well with the activity of fluconazole. The compounds $4 \mathrm{a}$ and $4 \mathrm{~d}$ also possess promising antifungal activity.

Minimum inhibition concentration (MIC)

The minimal inhibitory concentrations (MIC $50 \& 90$ ) of the strongly active compounds were also measured. The MICs of the extracts were determined by broth dilution method 
according to Alade and Irobi ${ }^{32}$ with a little modification. The extracts were serially diluted with normal saline $(0.9 \%)$ to $5-50 \mathrm{mg} / \mathrm{ml}$ preparation dispensed $(1.0 \mathrm{ml})$ into test tubes containing $1.0 \mathrm{ml}$ of nutrient or potato dextrose both. Each sensitive bacterial or fungal isolate $(100 \mu \mathrm{l})$ was inoculated into the test tubes. The tubes were mixed, covered with cotton wool and incubated at $37^{\circ} \mathrm{C}$ for bacteria and $25^{\circ} \mathrm{C}$ for fungi. Thereafter, the tubes were then examined for microbial growth.

Table 3: Antifungal activity of Chalcones and Pyrazoline derivatives.

\begin{tabular}{|c|c|c|c|}
\hline \multicolumn{3}{|c|}{ Antifungal Activity of Synthetic compound (In mm) } \\
\hline \multirow{2}{*}{ Compounds } & \multicolumn{3}{|c|}{ Organisms } \\
\cline { 2 - 4 } Flucanazole & A & B & C \\
\hline 3a & ++ & +++ & ++ \\
\hline 3b & + & ++ & + \\
\hline $\mathbf{3 c}$ & + & ++ & ++ \\
\hline $\mathbf{3 d}$ & + & ++ & + \\
\hline $\mathbf{3 e}$ & + & ++ & + \\
\hline $\mathbf{4 a}$ & ++ & ++ & + \\
\hline $\mathbf{4 b}$ & + & +++ & +++ \\
\hline $\mathbf{4 c}$ & +++ & +++ & +++ \\
\hline $\mathbf{4 d}$ & ++ & ++ & +++ \\
\hline $\mathbf{4 e}$ & + & ++ & +++ \\
\hline \multicolumn{4}{|c|}{ A: $\mathbf{M u c o r}, \mathbf{B}: \mathbf{P e n i c i l l i u m}, \mathbf{C}:$ Aspergillus } \\
\hline \multicolumn{4}{|c|}{} \\
\hline
\end{tabular}

Table 4: Minimum inhibitory concentration (MIC) values (MIC50 \& MIC90) of Most Potent compounds measured on standard bacterial strains.

\begin{tabular}{|c|c|c|c|c|c|c|c|c|c|c|}
\hline \multicolumn{6}{|c|}{$\begin{array}{c}\begin{array}{c}\text { MIC50 of Antibacterial Agent } \\
(\mathrm{mg} / \mathrm{ml})\end{array} \\
\end{array}$} & \multicolumn{5}{|c|}{ MIC90 of Antibacterial Agent (mg/ml) } \\
\hline \multirow{2}{*}{ 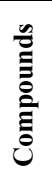 } & \multicolumn{5}{|c|}{ Organisms } & \multicolumn{5}{|c|}{ Organisms } \\
\hline & $\mathbf{A}$ & C & D & F & G & $\mathbf{A}$ & $\mathbf{C}$ & D & $\mathbf{F}$ & G \\
\hline $3 \mathbf{b}$ & 755.86 & 1060.45 & - & - & - & 1360.54 & 1908.80 & - & - & - \\
\hline 3d & 853.24 & - & - & - & - & 1535.84 & - & - & - & - \\
\hline $3 e$ & - & 1184.83 & - & 1492.54 & - & - & 2132.70 & - & 2686.57 & - \\
\hline $4 \mathrm{~b}$ & 445.43 & 345.18 & & - & - & 801.78 & 621.33 & - & - & - \\
\hline 4d & 439.37 & - & 874.89 & - & 380.23 & 790.86 & - & 1574.80 & - & 684.41 \\
\hline $4 \mathrm{e}$ & - & - & - & 644.75 & 646.41 & - & - & - & 1160.54 & 1163.54 \\
\hline 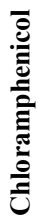 & 340.14 & 327.65 & 339.90 & 481.00 & - & 612.24 & 589.78 & 611.83 & 865.80 & - \\
\hline
\end{tabular}


Table 5: Minimum inhibitory concentration (MIC) values (MIC50 \& MIC90) of Most Potent compounds measured against three fungi strains.

\begin{tabular}{|c|c|c|c|c|c|c|}
\hline \multirow{2}{*}{ Compounds } & \multicolumn{3}{|c|}{$\begin{array}{c}\text { MIC 50 of Antifungal Activity } \\
\text { (mg/ml) }\end{array}$} & \multicolumn{3}{c|}{$\begin{array}{c}\text { MIC 90 of Antifungal Activity } \\
\text { (mg/ml) }\end{array}$} \\
\cline { 2 - 7 } & \multicolumn{3}{|c|}{ Organisms } & \multicolumn{3}{c|}{ Organisms } \\
\cline { 2 - 7 } & A & B & C & A & C \\
\hline Fluconazole & 206.27 & 196.62 & 241.2 & 371.28 & 353.9 & 434.15 \\
\hline 3a & 1650 & 248.23 & 878.57 & 2970 & 446.8 & 1581.4 \\
\hline 3b & 611.11 & 296.61 & 323.68 & 1100 & 533.9 & 582.63 \\
\hline 3c & 423.08 & 321.1 & 455.56 & 761.54 & 578 & 820 \\
\hline 3d & 785.71 & 307.02 & 1537.5 & 1414.3 & 552.6 & 2767.5 \\
\hline 3e & 206.25 & 241.38 & 512.5 & 371.25 & 434.5 & 922.5 \\
\hline 4a & 611.11 & 197.74 & 192.19 & 1100 & 355.9 & 345.94 \\
\hline 4b & 196.43 & 191.26 & 189.23 & 353.57 & 344.3 & 340.62 \\
\hline 4c & 279.66 & 207.1 & 189.23 & 503.39 & 372.8 & 340.62 \\
\hline 4d & 445.95 & 234.9 & 180.88 & 802.7 & 422.8 & 325.59 \\
\hline 4e & 208.86 & 192.31 & 192.19 & 375.95 & 346.2 & 345.94 \\
\hline \multicolumn{7}{|c|}{ A: Mucor, B: Penicillium, C:Aspergillus niger } \\
\hline
\end{tabular}

\section{Conclusions}

All compounds were screened for their in vitro antifungal against mucor, aspergillus and pecillium and in vitro antibacterial against Bacillus megaterium, Bacillus subtilus, Micrococcus luteus, Staphylococcus aureus, Eschericha coli, Enterobacter, Proteus vulgaris and Pseudomonas aeruginosastrains. Zone of inhibition were measured in millimeters. The antifungal activities of the tested compounds were compared with standard drug fluconazole. DMF was used as solvent. Compound $4 \mathrm{~b}, 4 \mathrm{~d}$ and $4 \mathrm{e}$ found most active against all three fungi compared to standard drug. Chloramphenicol was used as standard drugs for antibacterial activity. The compounds $4 \mathrm{~b}, 4 \mathrm{~d}$ and $4 \mathrm{e}$ showed significant activity against Bacillus megaterium, Bacillus subtilus, Micrococcus luteus, Staphylococcus aureus, Eschericha coli, Enterobacter, Proteus vulgaris and Pseudomonas aeruginosa strains.

\section{Acknowledgements}

The author's wishes to express their thanks to, Principal, V.P. \& R.P.T.P. Science College, the Head and Staff of Industrial Chemistry Department for providing laboratory facility.

\section{References}

1. Mohamed A M, Magdy M G, Magda N N, Waleed A H B, Arch.Pharm. Pharm. Med. Chem. 2004, 337, 427.

2. Andriote V T, J. Antimicrob. Chemother. 1999, 44, 151.

3. Silverman R B, Organic Chemistry of Drug Design and Drug Action, Academic Press, San Diego, 1992.

4. Thompson L A, Ellman J A, Chem. Rev. 1996, 96, 555.

5. Ali M A, Shaharyar M, Siddiqui A A, Sriram D, Yogeeswari P, Clercq E D, Acta Pol. Pharma. Drug Res. 2007, 63, 435.

6. Shaharyar M, Siddiqui A A, Ali M A, Bioorg. Med. Chem. Lett. 2006, 16, 4571. 
7. Chimenti F, Bizzarri B, Manna F, Bolasco A, Secci D, Chimenti P, Granese A, Rivanera D, Lilli D, Scaltritoc M M, Brenciaglia M I, Bioorg. Med. Chem. Lett. 2005, 15, 603.

8. Prasad Y R, Rao A L, Prasoona K, Murali K, Kumar P R, Bioorg. Med. Chem.Lett. 2005, 15, 5030.

9. Parmar S S, Pandey B R, Dwivedi C, Harbinson R D, J. Pharm. Sci. 1974, 63,1152.

10. Soni N, Pande K, Kalsi R, Gupta T K, Parmar S S, Barthwal J P, Res. Commun.Chem. Pathol. Pharmacol. 1987, 56, 129.

11. Batulin Y M, Farmakol Toksikol 1968, 31, 533.

12. Turan-Zitouni G, Chevallet P, Kilic F S, Erol K, Eur. J. Med. Chem. 2000, 35, 635.

13. Jeong T-S, S KimKim J-R K, Cho K-Y, Lee S, Bioorg. Med. Chem. Lett. 2004, 14, 2719.

14. Johnson M, Brent Younglove B, Lee L, LeBlanc R, Holt Jr H, Hills P, Mackay H, Brown T, Mooberry S L, Lee M, Bioorg. Med. Chem. Lett. 2007, 17, 5897.

15. Manna F, Chimenti F, Fioravanti R, Bolasco A, Secci D, Chimenti P, Ferlinib C, Scambia G, Bioorg. Med. Chem. Lett. 2005, 15, 4632.

16. Roecker A J, Coleman P J, Mercer S P, Schreier J D, Buser C A, Walsh E S, Hamilton K, Lobell R B, Tao W, Diehl R E, South V J, Davide J P, Kohl N E, Yan Y, Kuo L C, Li C, Fernandez-Metzler C, Mahan E A, Prueksaritanontd T, Hartmana G D, Bioorg. Med. Chem. Lett. 2007, 17, 5677.

17. Puig-Basagoiti F, Tilgner M, Forshey B M, Philpott S M, Espina N G, Wentworth D E, Goebel S J, Masters P S, Falgout B, Ferguson P R D M, Shi P-Y, Antimicrob. Agents Chemother. 2006, 50, 1320.

18. Ali M A, Shaharyar M, Siddiqui A A, Sriram D, Yogeeswari P, Clercq E D, Acta Pol. Pharma. Drug Res. 2007, 63, 423.

19. Heindel N D, Reid J R, J. Heterocycl. Chem. 1980, 17, 1087.

20. Holla B S , Kalluraya B, Sridhar K R, Drake E, Thomas L M, Bhandary K K, Levine M S, Eur. J. Med. Chem. 1994, 29, 301.

21. Mathew V, Keshavayya J, Vidya V P, Acharya, Reddy B M, Eur. J.Med. Chem. 2006, 41, 1048.

22. The Merck Index, Merck Co. Inc., twelfth ed., USA, 1996.

23. Haber J, Present status and perspectives on antimycoties with systematic effects, Cas. Lek. Cesk. 2001, 140, 596.

24. Brucato A, Coppola A, Gianguzza S, Provenzano P M, Boll. Soc. Ital.Biol. Sper. 1978, 54, 1051.

25. Coffen D L, Fryer R I, U.S. Patent 1974, 3, 849, 434; Chem. Abstr. 1975, 82, 73044v.

26. Shiroki M, Tahara T, Araki K, Jap. Patent 1975, 75100096; Chem. Abstr. 1976, 84, $59588 \mathrm{k}$.

27. Povelitsa F D, Gural A G, Antibiotiki Moscow 1973, 18, 71; Chem. Abstr. 1973, 78, 93044.

28. Panchal A D, Patel P M, E-Journal of Chemistry. 2011, 8(3), 1180-1185.

29. Barry A L, In A. L. Barry (ed.), Lea \& Febiger, Philadelphia. 1976, 163.

30. Cooper K E, In F. Kavanagh (ed.), Analytical microbiology II. New York. 1972, 13.

31. Mallette M F, Norris J R, and Ribbons D W, Methods in Microbiology. 1969, 1, 521.

32. Alade P I, Irobi O N, Journal of Ethnopharmacology. 1993, 39(3), 171. 


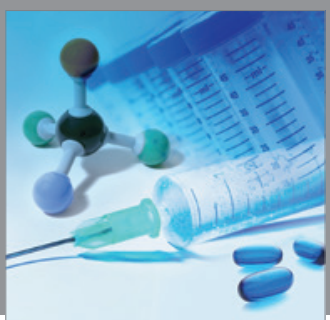

International Journal of

Medicinal Chemistry

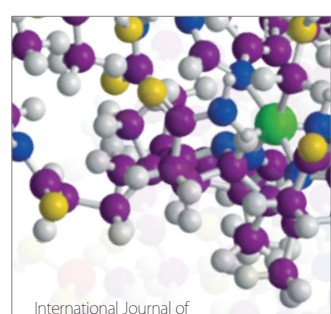

Carbohydrate Chemistry

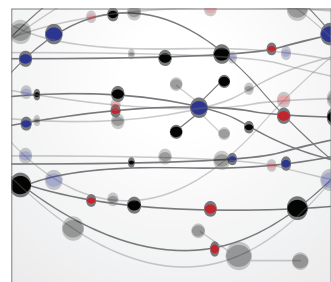

The Scientific World Journal
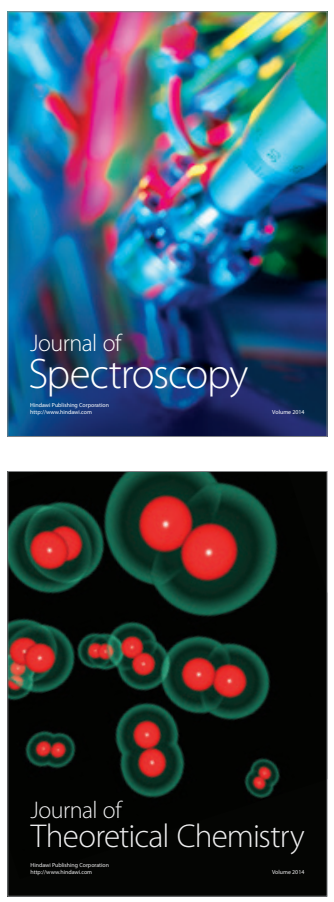
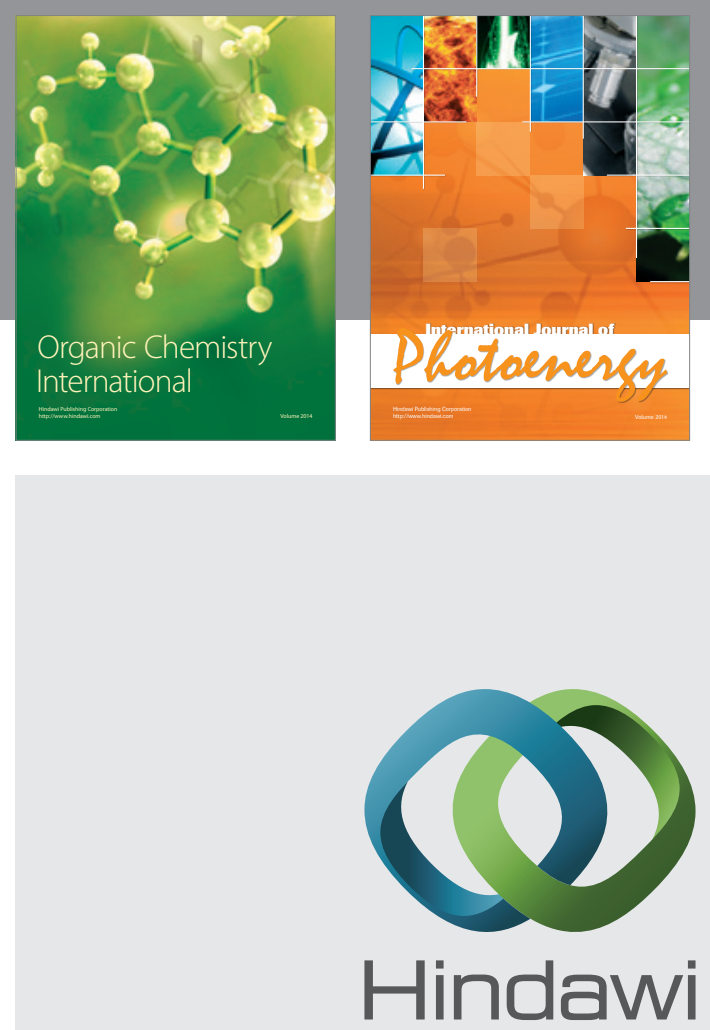

Submit your manuscripts at

http://www.hindawi.com
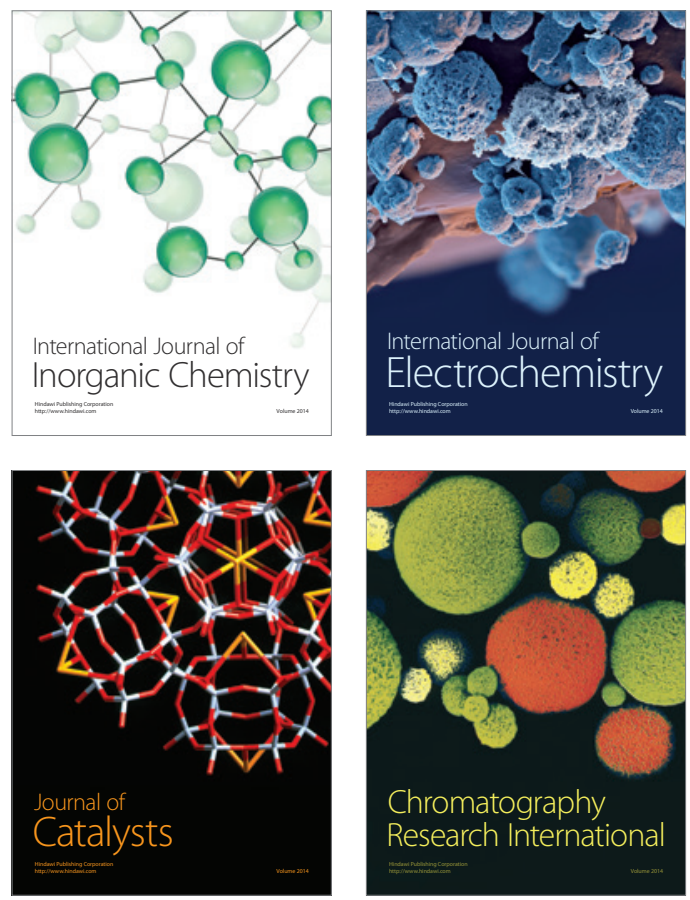
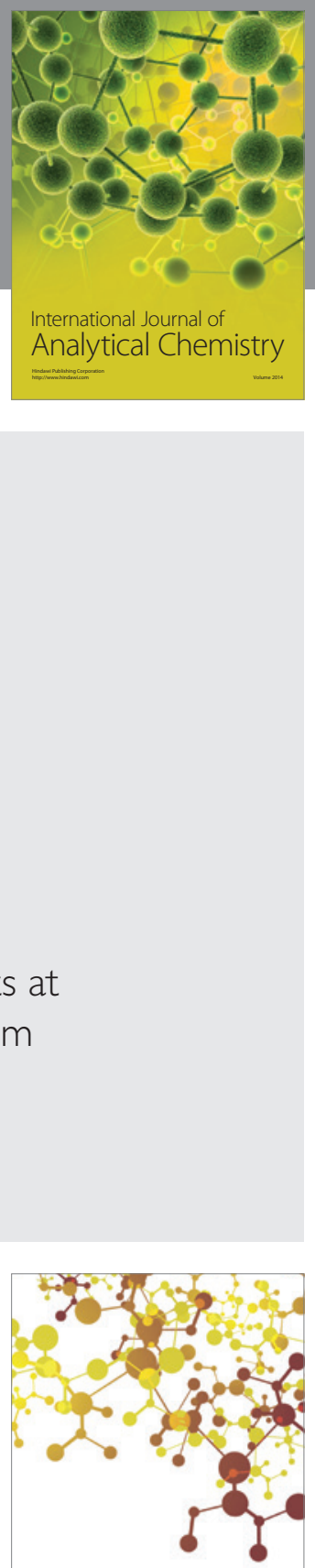

Journal of

Applied Chemistry
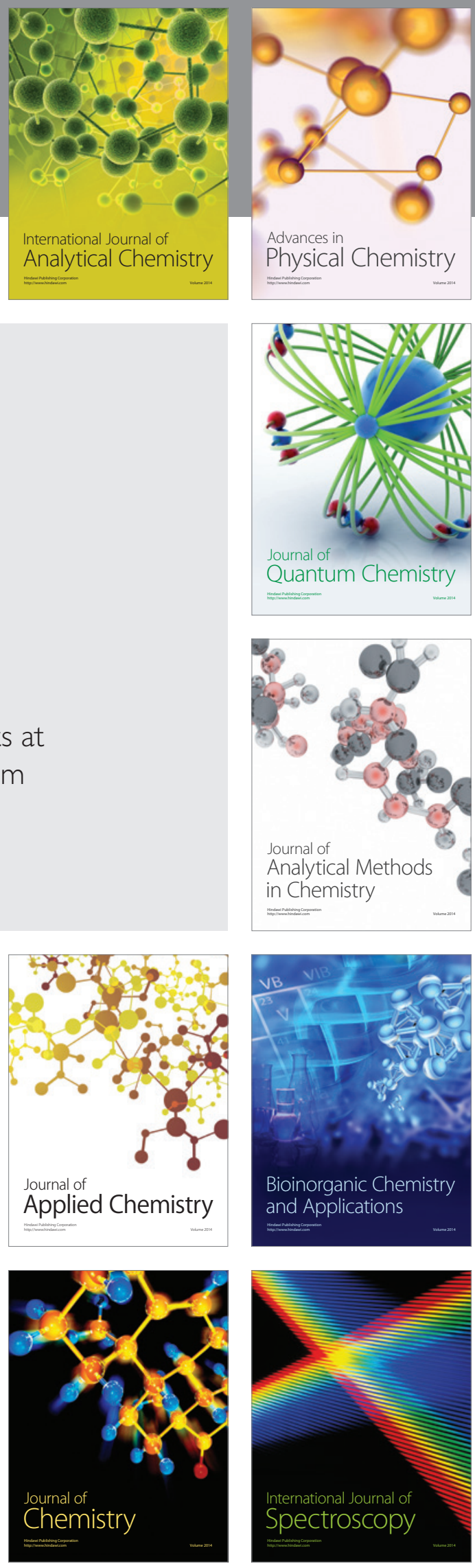